\title{
Simulation and Study on Non-Sinusoidal Oscillation Control System of Continuous Casting Mold
}

\author{
Xiaojun Zhang
}

School of Mechanical Engineering and Automation, University of Science and Technology LiaoNing, Anshan China 114051

myfriendjun@163.com

Keywords: mold oscillation, hydraulic servo control system, non- sinusoidal, simulation

\begin{abstract}
The paper describes the composition and working principle of the mold oscillation control system. The mathematical model of hydraulic servo control system is established. The total system transfer function is established by MATLAB program. By using the SIMULINK module, we determined various components' parameter values. We got different simulation curves and Bode plots by inputting non- sinusoidal function and carrying out the dynamic simulation to the mold oscillation mathematical model, we also analyzed stability of the whole system to verify the accuracy of system design.
\end{abstract}

\section{Introduction}

Mold oscillation is a basic feature to continuous casting. After using oscillatory mold, continuous casting achieved industrial production in the history of continuous casting [1]. With the development of high-speed caster, casting speed is faster and faster, but high speed casting decreases the lubrication performance between mold and casting blank, and it makes casting blank produce adhesive breaking out. Non-sinusoidal oscillation must be used to solve the problem above, in addition to using new type protecting slag. At present, we usually use hydraulic servo control system to generate the non-sinusoidal oscillation.

\section{System Description}

Mold oscillation system (as shown in Fig.1) consists of mold, vibration table, hydraulic cylinder, buffer spring, vibration base and so on.

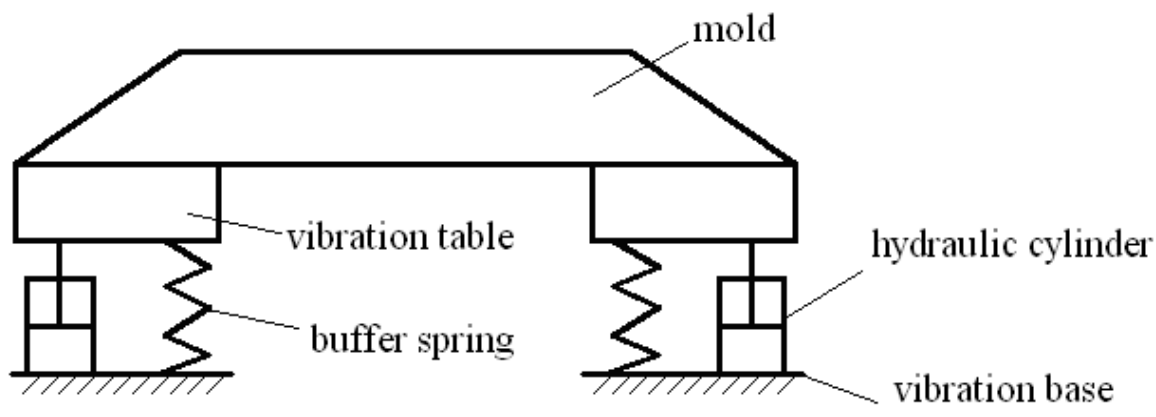

Fig. 1 Diagram of system composition

Mold and vibration table are connected together by using the bolts. The hydraulic cylinder lift vibration table up to realize alternating motion of the mold in vertical direction. Position of piston rod of hydraulic cylinder is fed back to the computer by displacement sensor. Error signal can be got by comparing with the given signal. And then the computer drive servo valve to control hydraulic cylinder motion by PID regulation [2, 3]. So, a position closed-loop control system is constituted as shown in Fig.2. 


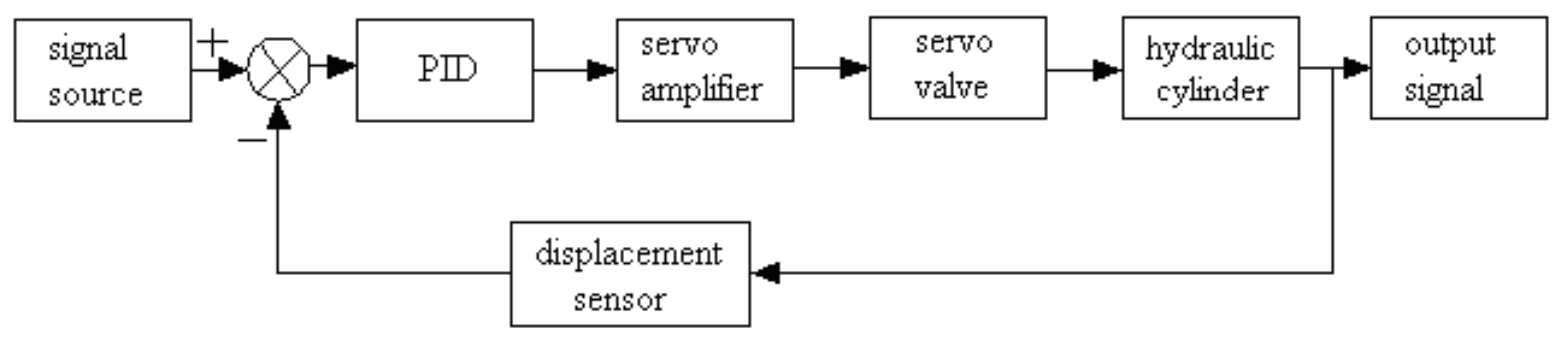

Fig. 2 Block-diagram of control system

\section{Establishment of Transfer Function}

Transfer functions of servo amplifier and displacement sensor can be simplified proportional element, because corner frequencies of them are much higher than System Bandwidth [4]. And then, it is important in thinking about how to establish mathematical models of electro-hydraulic servo valve and hydraulic cylinder.

Electro-hydraulic Servo Valve. Usually, the electro-hydraulic servo valve can be approximate as a second-order oscillation system. Its transfer function is as follows.

$$
G_{s v}(s)=\frac{K_{s v}}{\frac{s^{2}}{\omega_{s v}^{2}}+\frac{2 \zeta_{s v}}{\omega_{s v}} s+1}
$$

where, $K_{s v}$ is static flow gain of servo valve, and $\omega_{s v}$ is natural frequency of servo valve. $\zeta_{s v}$ is damping ratio of servo valve.

Hydraulic Cylinder. Generally, when eliding elastic load in the project, executive component and controlled member are regarded as a combination of integral and second-order systems. Its transfer function is as follows.

$$
H(s)=\frac{1 / A_{h}}{s\left(\frac{s^{2}}{\omega_{h}^{2}}+\frac{2 \zeta_{h}}{\omega_{h}} s+1\right)} .
$$

where, $A_{h}$ is effective area of hydraulic cylinder, and $\omega_{h}$ is hydraulic natural frequency of hydraulic cylinder system. $\zeta_{h}$ is comprehensive damping ratio of hydraulic cylinder system.

Open-loop Transfer Function of System. According to the following system parameters such as oil density, dynamic viscosity, oil compressibility, inside diameter of hydro-cylinder, diameter and effective area of piston rod etc., hydraulic natural frequency of system can be calculated to confirm transfer function of all parts of system. So, open-loop transfer function of system is as follows.

$$
G(s)=\frac{0.03 \times 0.005 \times 250 \times 240}{\left(\frac{1}{380^{2}} s^{2}+\frac{2 \times 0.6}{380} s+1\right)\left(\frac{1}{290^{2}} s^{3}+\frac{2 \times 0.15}{290} s^{2}+s\right)}
$$

\section{Dynamic Simulation in MATLAB}

The non-sinusoidal input function can be constructed by MATLAB program, as shown in Fig. 3. It shows that total fall time is less than total rise time. That is, negative strip time decreases and positive strip time increases to effectively improve casting speed and reduce chatter mark of casting blank surface [5]. 


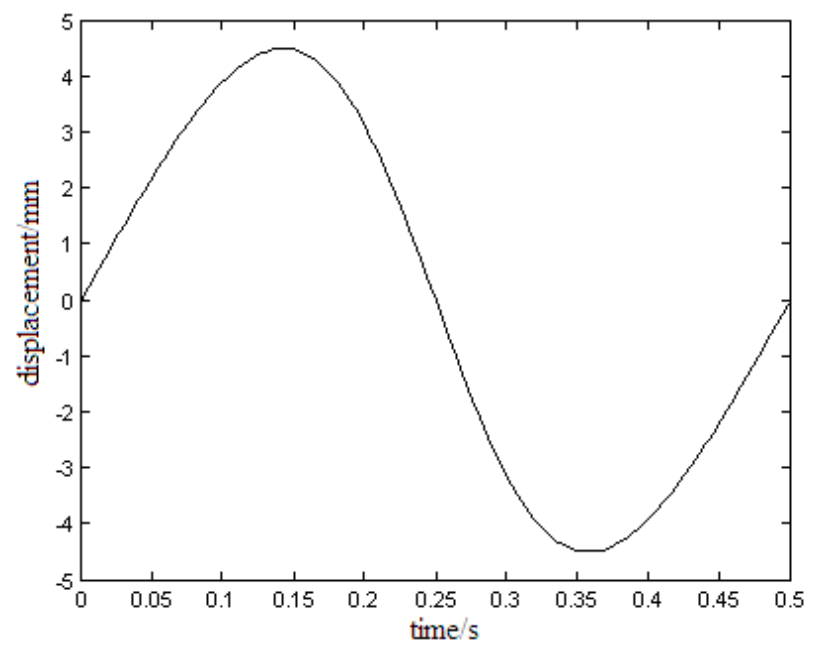

Fig. 3 The curve of non-sinusoidal function

According to Fig.2, the corresponding modules are called in SIMULINK, and double-click on each module, fill in above found parameter and then connect every module into block-diagram, as shown in Fig.4.

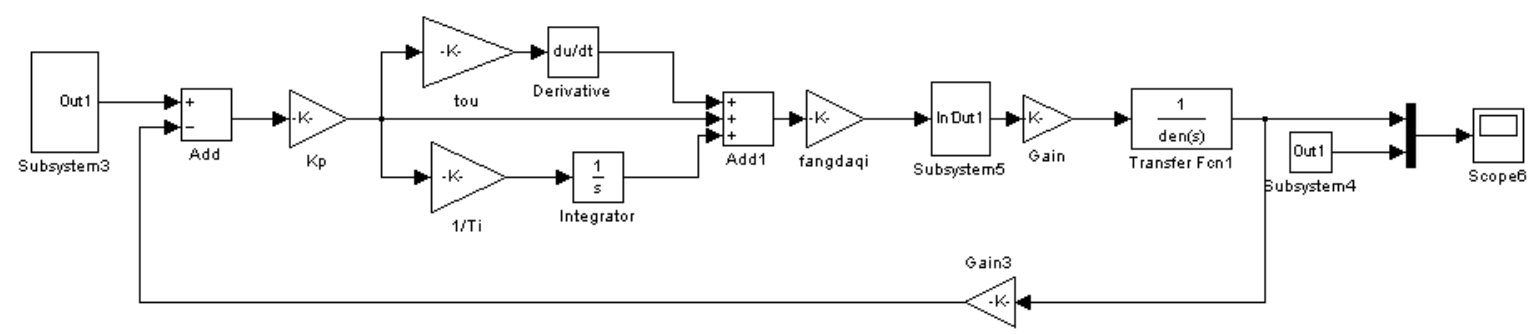

Fig. 4 Block-diagram of control system in MATLAB

In order to make contrast, the non-sinusoidal signals that have same amplitude, different frequency are input system to simulate. Accordingly, simulating curves are obtained as shown in Fig. 5. Looking from the simulation results, the output waveform and the input one are very similar, except for initial simulation stage. Comparing Fig. 5a with Fig.5b, we can see that the bigger frequency is, the longer time of response delay is. Whether high or low frequency, 1.2 seconds later input and output signals have the same waveform, and phase delay is very small. It indicates that output signal has very good follow ability to input signal and operation of the system is stable and reliable.

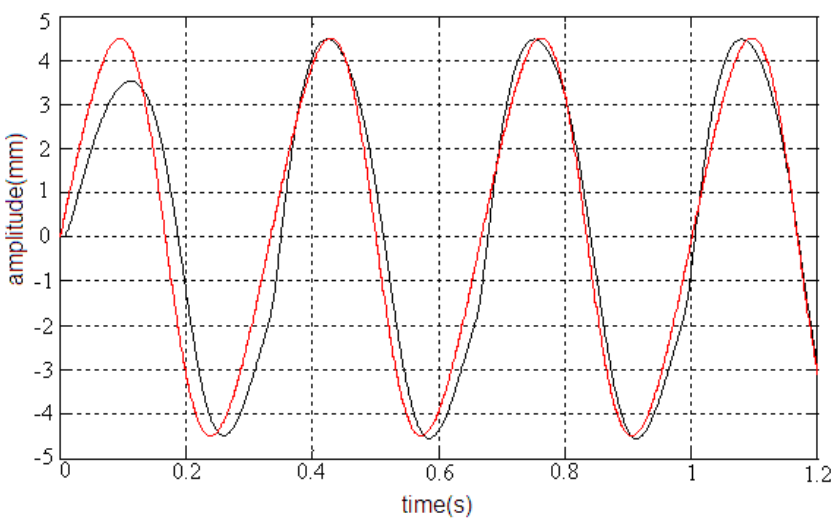

(a)

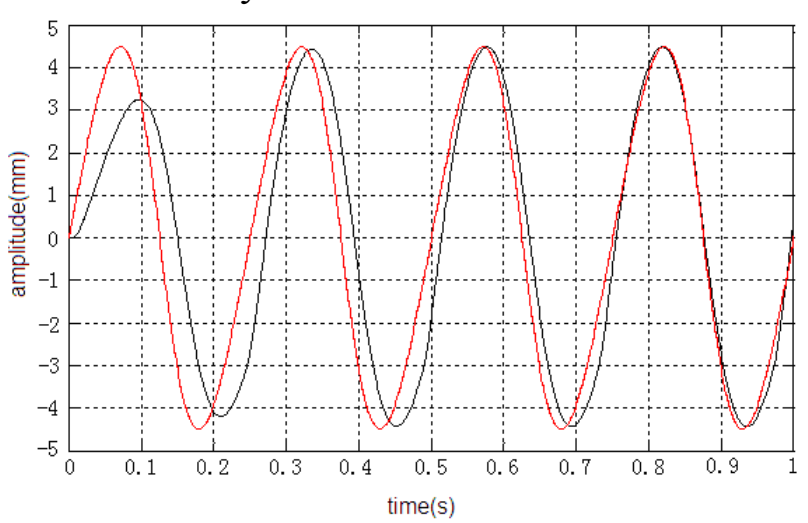

(b)

Fig. 5 Simulation curve of control system

(a) $\mathrm{f}$ (frequency) $=3 \mathrm{~Hz}, \mathrm{~A}$ (amplitude) $=4.5 \mathrm{~mm}$, (b) $\mathrm{f}=4 \mathrm{~Hz}, \mathrm{~A}=4.5 \mathrm{~mm}$ Bode plot of overall system is obtained by MATLAB program as shown in Fig. 6, according to the transfer function that Eq. 3 described. It can be seen from the figure that the system is stable too. 


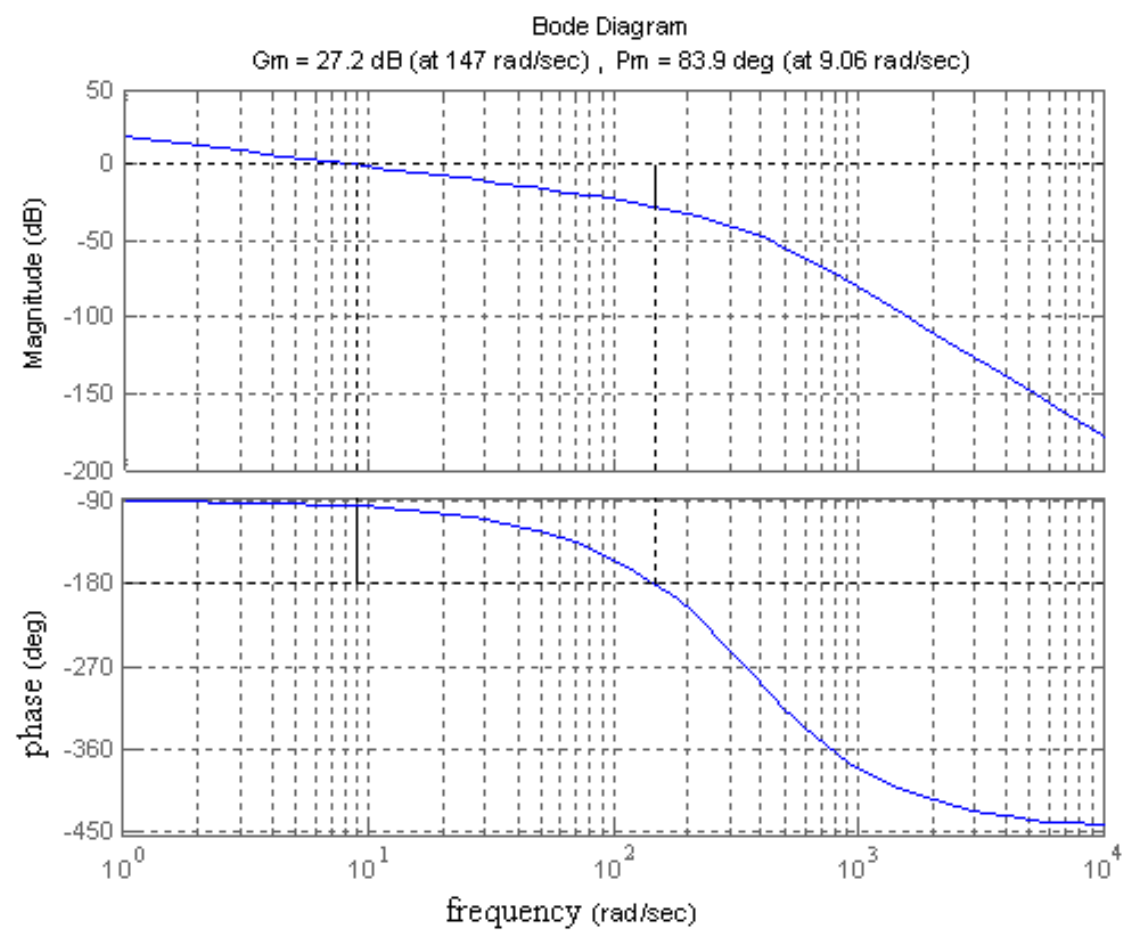

Fig. 6 Bode plot of control system

\section{Conclusion}

We established the mathematic model of mold oscillation control system and carried out the simulation by using SIMULINK program. In order to verify the correctness of the design, we analyzed the stability and responsiveness of system. Through the simulation of control system, we understood that parameter's change has an effect on responsibility of system. It provides a reliable theoretical basis for further study on control system of mold oscillation.

\section{References}

[1] Li Xian-kui, Zhang De-ming, Continuous casting mould oscillation technology, Metallurgical Industry Press, Beijing, 2000.

[2] HU Jun-hong, ZHOU Ya-jun, Research of mold electro- hydraulic servo oscillation system on Baosteel continuous casting testing platform, Metallurgical Industry Automation, No. 6(2005), 6-10.

[3] ZHANG Dong, MENG You-ping, Simulation for hydraulic oscillating systems of cast machine mould based on MATLAB, Fluid Power Transmission and Control, No.2 (2008), 30-31.

[4] Song Zhi-an, Analysis and design for hydraulic servo system based on MATLAB, National Defence Industry Press, Beijing, 2007.

[5] YUAN Si-cong, Guo Jin-ping, JI Jun, Modeling and dynamic simulation for mould crystallizer oscillation based on ADAMS, Coal Mine Machinery, Vol.28 No.3( 2007), 44-47. 